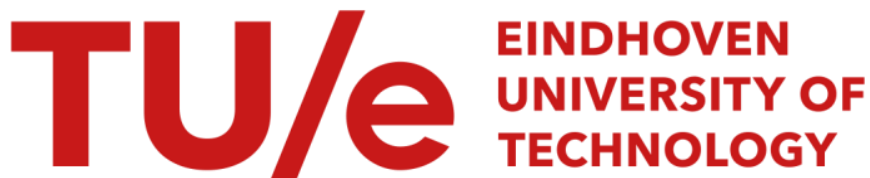

\section{Bayesian reliability analysis with imprecise prior probabilities}

Citation for published version (APA):

Coolen, F. P. A., \& Newby, M. J. (1994). Bayesian reliability analysis with imprecise prior probabilities. Reliability Engineering and System Safety, 43, 75-85. https://doi.org/10.1016/0951-8320\%2894\%2990096-5, https://doi.org/10.1016/0951-8320(94)90096-5

DOI:

10.1016/0951-8320\%2894\%2990096-5

10.1016/0951-8320(94)90096-5

Document status and date:

Published: 01/01/1994

\section{Document Version:}

Publisher's PDF, also known as Version of Record (includes final page, issue and volume numbers)

\section{Please check the document version of this publication:}

- A submitted manuscript is the version of the article upon submission and before peer-review. There can be important differences between the submitted version and the official published version of record. People interested in the research are advised to contact the author for the final version of the publication, or visit the $\mathrm{DOI}$ to the publisher's website.

- The final author version and the galley proof are versions of the publication after peer review.

- The final published version features the final layout of the paper including the volume, issue and page numbers.

Link to publication

\section{General rights}

Copyright and moral rights for the publications made accessible in the public portal are retained by the authors and/or other copyright owners and it is a condition of accessing publications that users recognise and abide by the legal requirements associated with these rights.

- Users may download and print one copy of any publication from the public portal for the purpose of private study or research.

- You may not further distribute the material or use it for any profit-making activity or commercial gain

- You may freely distribute the URL identifying the publication in the public portal.

If the publication is distributed under the terms of Article 25fa of the Dutch Copyright Act, indicated by the "Taverne" license above, please follow below link for the End User Agreement:

www.tue.nl/taverne

Take down policy

If you believe that this document breaches copyright please contact us at:

openaccess@tue.nl

providing details and we will investigate your claim. 


\title{
Bayesian reliability analysis with imprecise prior probabilities
}

\author{
F. P. A. Coolen ${ }^{a}$ \& M. J. Newby ${ }^{b}$ \\ ${ }^{a}$ Department of Mathematics and Computing Science, 'Department of Industrial Engineering and Management Science, and \\ Frits Philips Institute for Quality Management, Eindhoven University of Technology, PO Box 513, 5600MB Eindhoven, \\ The Netherlands
}

(Received 9 December 1992; accepted 24 May 1993)

\begin{abstract}
The Bayesian framework for statistical inference offers the possibility of taking expert opinions into account, and is therefore attractive in practical problems concerning the reliability of technical systems. Probability is the only language in which uncertainty can be consistently expressed, and this requires the use of prior distributions for reporting expert opinions. In this paper an extension of the standard Bayesian approach based on the theory of imprecise probabilities and intervals of measures is developed. It is shown that this is necessary to take the nature of experts' knowledge into account. The application of this approach in reliability theory is outlined. The concept of imprecise probabilities allows us to accept a range of possible probabilities from an expert for events of interest and thus makes the elicitation of prior information simpler and clearer. The method also provides a consistent way for combining the opinions of several experts.
\end{abstract}

\section{INTRODUCTION}

Reliability analysis is concerned with the ability of a system to function satisfactorily under a given set of circumstances, and thus gives important information about the quality of a system, when to replace it, or when and how to maintain it. Many probabilistic models for the reliability of components and systems are available and can be used without too much difficulty whenever the parameters of the system are given or are deducible from the system's properties. The situation is more difficult when parameters must be estimated from data. The use of models is often limited by a lack of satisfactory data on which estimates of system parameters can be based. The reasons for the lack of data are numerous; sometimes there is no effective registration system, sometimes the data are corrupted, and sometimes changes in design or operating conditions mean that historical data are no longer directly relevant.

The Bayesian approach to statistical inference ${ }^{1}$ provides a framework for dealing with expert opinions and prior knowledge together with historical or experimental data. Bayesian methods therefore offer solutions to some of the difficulties in reliability analysis. ${ }^{2,3}$ However, there are also difficulties in using probability as language to express expert opinions. ${ }^{4}$ The problem has two aspects: the elicitation of expert knowledge, and the expression of that knowledge in the form of a prior distribution. Within the standard probability framework combined with the Bayesian approach it is difficult to deal with the uncertainty of an expert who says, for example, 'I think the chance of failure is between $25 \%$ and $35 \%$ '. To deal with this uncertainty we can resort to hierarchical models, but they simply push the problem to another level. Sensitivity analyses can also be used to examine the effect of the prior on the posterior and predictive distributions. In situations where there are plenty of good-quality data available these options are likely to yield reasonable results because the evidence from the data weighs so much more heavily than the prior evidence.

A further difficulty arises when precise knowledge about some possible events exists besides ignorance about other events. This situation demands a prior exactly specified in only a part of the sample space.

A second area of difficulty is that of combining expert opinions and knowledge for use in decisionmaking. The Kolmogorov axioms require exact probabilities, and do not provide a basis for reporting 
agreement or disagreement between experts. Within the Bayesian framework this can be solved by constructing the natural Bayesian weights or by using a hierarchical model in which the decision-maker also has a prior for the experts. ${ }^{5,6}$ However again within the Bayesian framework at some level in the model a specified prior must be chosen.

Thus a calculus for consistent decision-making is required within which the decision-maker or expert does not need to enter the realm of probabilities in those cases where probability remains a mystery. Moreover, a calculus is required which is not restricted by the fact that most people cannot give a consistent estimation of a set of probabilities.

In the literature there is a growing interest for non-standard probability theories to deal with, for example, reliability problems. ${ }^{7-9}$ This is an indication that the standard probability theory fails at some points. Most of these new techniques use some kind of higher order probabilities, often with some vague name (membership functions, possibility) and vague interpretation. The theory described in this paper generalizes the standard probability theory by dropping an assumption that is too strong in practical application, as is described in Section 2. The theory, of course, needs to be evaluated by practical application. We believe that one should not ask more from experts than some statements about observables, such as time to failure, and certainly not possibility distributions for precise probabilities, as the experts will have enough problems understanding probability itself.

In Section 2 of this paper, the concept of imprecise probabilities is briefly discussed in the context of reliability theory. In Section 3 Bayesian analysis with imprecision, the elicitation and combination of experts' opinions, are discussed, and in Section 4 two examples of applications to reliability problems are given. Finally, in Section 5 the conclusions of this paper are summarized and some additional remarks are made.

\section{IMPRECISE PROBABILITIES AND RELIABILITY}

In this section the definitions of the measures of interest in reliability analysis are extended in a consistent way using imprecise probabilities expressed as imprecise prior distributions in a Bayesian analysis.

The Bayesian approach to statistical inference demands a prior distribution for the parameters of interest. The prior represents the information available before actual data come available. Walley ${ }^{4}$ presents a clear and extensive discussion about the major difficulties involved in indicating the amount of information, or lack of information, on which a prior distribution is based. When the prior information consists of opinions of several experts, it is important for the decision-maker to report the level of disagreement among the experts. This is not possible with a single distribution.

To deal with the above shortcomings the concept of imprecise probabilities is introduced, ${ }^{4}$ where instead of one value for the probability $\mathscr{P}(A)$ of an event $A$, an interval of values is chosen, bounded by lower and upper probabilities for $A$. This interval can be interpreted as a set of possible values for $\mathscr{P}(A)$, between which no further distinction can be made given the information available. This implies that these bounds depend on you and your information, and therefore have a subjective nature.

This idea suggests that small intervals arise when there is plenty of relevant information, and larger intervals when there is a lack of information. Total lack of information about the probability of an event can be represented by assigning the values 0 and 1 to the lower and upper probabilities for this event. Walley ${ }^{4}$ uses an interpretation related to the subjective nature of probability as advocated by De Finetti, ${ }^{10}$ and it is this interpretation that makes clear the advantage of the theory compared to standard probability theory. Let a bet on event $A$ be such that the buyer of the bet receives 1 (unit of utility, see Walley, ${ }^{4}$ section 2.2) from the seller if $A$ occurs, and 0 otherwise. The lower probability $\mathscr{P}(A)$ for $A$ is defined as the supremum of all prices at which you are willing to buy this bet, and the upper probability $\overline{\mathscr{P}}(A)$ as the infimum of all prices at which you are willing to sell the bet, assuming that you only want to buy or sell if you expect a profit. This means that for prices in the interval $[\mathscr{\mathscr { P }}(A), \overline{\mathscr{P}}(A)]$ you are neither willing to buy nor to sell the bet, as you are not sure about your expectation of profit or loss at such prices. This models your lack of perfect knowledge about the probability $\mathscr{P}(A)$.

In standard Bayesian theory the lower and upper probabilities for $A$ are always equal (the probability for event $A$ is called 'precise'). This implies that two experts will always be prepared to exchange a bet (so have a conflict) about $A$, except if their precise probabilities are identical. This assumption is too strong and leads to the loss of important information.

Boole $^{11}$ introduced an idea of imprecise probabilities, but little attention was paid to it for more than a century. More recently, Smith ${ }^{12}$ and Good ${ }^{13}$ contributed to the theory of imprecise probabilities. Dempster's ${ }^{14}$ approach is also interesting, but can lead to unreasonable results in some cases (see Walley, ${ }^{4}$ section 5.13). Walley and Fine ${ }^{15}$ and Wolfenson and Fine $^{16}$ derive important results for the theory of imprecise probabilities, and Walley and Fine $^{17}$ propose a generalization of the frequentist theory to include imprecision. When the imprecise probabilities 
refer to parameters the method of intervals of measure ${ }^{18}$ is useful, as in the Bayesian framework. ${ }^{19}$

Although many authors have contributed to the theory of imprecise probabilities during the last decades, statistical models based on imprecision that can be applied easily in practice have received little attention. Adopting imprecise probabilities within the Bayesian framework offers the possibility of dealing with different levels of ignorance. The theory proposed by Berger ${ }^{20,21}$ for robust Bayesian inference is similar to that discussed here, but there imprecision enters through sensitivity analyses, and not as a means of reporting important information itself.

The theory in this paper is fundamentally different to sensitivity analysis (Walley, ${ }^{4}$ section 5.9 ), and also different to the method of Meczarski and Zielinski, ${ }^{22}$ who restrict their argument to a set of precise prior distributions belonging to a parametric family. A review of theories for handling uncertainty is given by Walley, ${ }^{23}$ who compares the theory discussed here with other theories for measuring uncertainty in expert systems, e.g. fuzzy sets.

For an event $A, A \subseteq \mathbb{A}$ ( $\mathbb{A}$ the sample space), the lower and upper probabilities, $\mathscr{P}(A)$ and $\overline{\mathscr{P}}(A)$ respectively, satisfy the basic axioms given by Smith $^{12}$ (see also Wolfenson and Fine ${ }^{16}$ ). Special cases of imprecise probabilities are lower and upper cumulative distribution functions (CDF) $\mathscr{F}(x)$ and $\mathscr{F}(x)$ for a real random variable $x$. These are the lower and upper probabilities of the events $X \leq x$ for $x \in \mathbb{R}$, defined by $\mathscr{F}(x)=\mathscr{P}(X \leq x)$ and $\overline{\mathscr{F}}(x)=\overline{\mathscr{P}}(X \leq x)$.

Following DeRobertis and Hartigan, ${ }^{18}$ suppose there exist functions $\ell(x)$ and $u(x), 0 \leq \ell(x) \leq u(x)$ for all $x$, such that all functions $h, \ell \leq h \leq u$, can, after normalization, be regarded as probability density functions (PDF) for $X$. The functions $\ell$ and $a$ are called lower and upper density functions, and in this paper are assumed to be continuous and with nonzero and finite integrals. If lower and upper densities are given, then the lower and upper CDFs are (for $x \in \mathbb{R}$ )

$$
\mathscr{F}(x):=\left(1+\frac{\int_{x}^{\infty} u(\omega) \mathrm{d} \omega}{\int_{-\infty}^{x} \ell(\omega) \mathrm{d} \omega}\right)^{-1}
$$

and

$$
\overline{\mathscr{F}}(x):=\left(1+\frac{\int_{x}^{\infty} \ell(\omega) \mathrm{d} \omega}{\int_{-\infty}^{x} u(\omega) \mathrm{d} \omega}\right)^{-1}
$$

which provide lower and upper envelopes (the lower and upper bounds with smallest distance of all lower and upper bounds) of all CDFs that can be constructed from densities (after normalization) that lies between $\ell$ and $a$ (Walley, ${ }^{4}$ section 4.6). The continuity of $\ell$ and $u$ ensures the continuity of these lower and upper CDFs. In the rest we use the term density to mean a function lying between $\ell$ and $u$ which after normalization yields a probability density function (PDF).

The mathematical forms of the upper and lower densities are not particularly simple, but all the discussions can be carried out in terms of imprecise probabilities for $X \leq x$ alone.

The imprecision for an event $A$ is defined by $\Delta(A)=\overline{\mathscr{P}}(A)-\mathscr{P}(A)$, so $0 \leq \Delta(A) \leq 1$. The extreme values, 0 and 1 , occur only in the case of precision $(\mathscr{P}(A)=\overline{\mathscr{P}}(A))$ or vacuous probabilities $(\mathscr{P}(A)=0$ and $\overline{\mathscr{P}}(A)=1)$. A simple measure for the amount of information about $A$ in $\mathscr{P}$ and $\overline{\mathscr{P}}$ is: $I(A)=\Delta(A)^{-1}-1$ (Walley, ${ }^{4}$ section 5.3.7). This is zero if and only if the probabilities are vacuous, and is infinite if and only if the probability for $A$ is precise.

In reliability theory, the reliability function, hazard rate and hazard function are important characterizations of probability distributions, with the hazard rate providing a natural description of a failure process. We define lower and upper hazard rates and hazard functions within the theory of imprecise probabilities. We restrict the discussion to a non-negative real random variable $T \geq 0$ (time).

\section{Definitions}

Suppose continuous lower and upper densities $\ell$ and $u$ are specified for $T$. The corresponding imprecise CDFs $\mathscr{F}$ and $\bar{F}$ are given by (1). Obvious definitions of the lower and upper reliability functions are $(t \geq 0)$

$$
\mathscr{R}(t):=1-\overline{\mathscr{F}}(t) \text { and } \overline{\mathscr{R}}(t):=1-\mathscr{F}(t)
$$

The lower and upper hazard rates are defined by

$$
\underline{h}(t):=\frac{\ell(t)}{\int_{t}^{\infty} u(x) \mathrm{d} x} \text { and } \bar{h}(t):=\frac{u(t)}{\int_{t}^{\infty} \ell(x) \mathrm{d} x}
$$

These imprecise hazard rates are the tightest lower and upper envelopes of the set of all hazard rates of PDFs that are derived from normalization of densities between $\ell$ and $u$, which is easily proved by taking a function $g$ with $\ell(t) \leq g(t) \leq u(t)$ for all $t \geq 0$ and $C_{g}:=\int_{0}^{\infty} g(x) \mathrm{d} x$ the normalizing constant. The hazard rate corresponding to $\mathrm{PDF} g(t) / C_{g}$ is

$$
h_{g}(t)=\frac{g(t) / C_{g}}{\int_{t}^{\infty} g(x) / C_{g} \mathrm{~d} x}=\frac{g(t)}{\int_{t}^{\infty} g(x) \mathrm{d} x}
$$

and the fact that $\ell$ and $u$ are the lower and upper envelopes of all such $g$ makes clear that the above defined $\underline{h}$ and $\bar{h}$ are the lower and upper envelopes of all such $h_{g}$.

The lower and upper hazard functions are defined 
by

$$
\mathscr{H}(t):=-\ln (1-\mathscr{F}(t)) \text { and } \overline{\mathscr{H}}(t):=-\ln (1-\overline{\mathscr{F}}(t))
$$

It is again obvious that these are the lower and upper envelopes of all possible hazard functions relating to possible PDFs $g(t) / C_{g}$ as above, since the hazard function for such a PDF is $\mathscr{H}_{g}(t)=-\ln \left(1-\mathscr{F}_{g}(t)\right)$, with $\mathscr{F}_{g}$ the CDF corresponding to PDF $g(t) / C_{g}$ and $\mathscr{F}$ and $\mathscr{F}_{F}$ the lower and upper envelopes of all such $\mathscr{F}_{q}$.

Note that $h$ and $\bar{h}$ are not the first derivatives of $\mathscr{H}$ and $\overline{\mathscr{K}}$; in fact, $h(t) \leq \mathscr{H}^{\prime}(t)$ and $\bar{h}(t) \geq \mathscr{\mathscr { K }}^{\prime}(t)$ for all $t \geq 0$, with equality iff $\ell(t)=u(t)$.

\section{BAYESIAN ANALYSIS AND IMPRECISION}

We concern ourselves here with a situation where the probabilistic model for the failure mechanism is known, and moreover is described by a failure PDF $f_{x}(x \mid \theta)$. The form of the density is assumed to be known precisely, and the information that we obtain as prior opinions and as data enters the model through $\theta$. Thus our model is $f_{X}(x \mid \theta)$ and on the basis of data and prior opinion about $\theta$ we wish to make probability statements about $X$. Our prior knowledge is expressed imprecisely through imprecise lower and upper prior densities, $\ell(\theta)$ and $u(\theta)$, for $\theta$. For simplicity, $\theta$ and $X$ are assumed to be real numbers, but the restriction is not necessary.

Given a vector $\mathrm{x}=\left(x_{1}, \ldots, x_{n}\right)$ of $n$ independent observations, the likelihood function $\mathscr{L}(\theta \mid \mathbf{x})$ is determined and because $f_{x}$ is of fixed form, so is the likelihood. Thus the updated versions of $\ell(\theta)$ and $u(\theta)$ are (Walley, ${ }^{4}$ section 4.6) $\ell(\theta \mid \mathbf{x}):=$ $\mathscr{L}(\theta \mid \mathbf{x}) \mathcal{\ell}(\theta)$ and $u(\theta \mid \mathbf{x}):=\mathscr{L}(\theta \mid \mathbf{x}) u(\theta)$, bounding (up to a constant factor) all standard Bayesian posteriors related to prior PDFs $h$, that are normalized versions of functions between $\ell$ and $u$. It can be proved straightforwardly that the set of all PDFs that can be constructed by normalizing functions between these updated $\ell$ and $u$, is equal to the set of all posterior PDFs resulting from priors that follow by normalizing functions between the prior $\ell$ and $u$. The corresponding lower and upper posterior CDFs, $\mathscr{F}(\theta \mid \mathbf{x})$ and $\mathscr{F}(\theta \mid \mathbf{x})$, are derived as in (1).

Consistent definitions (in the sense that the above $\ell(\theta \mid \mathbf{x})$ and $u(\theta \mid \mathbf{x})$ are consistent definitions of the posterior imprecise densities) of lower and upper predictive densities are:

$$
\ell_{X}(x):=\int_{-\infty}^{\infty} \ell_{X}(x \mid \theta) \ell(\theta) \mathrm{d} \theta
$$

and

$$
u_{X}(x):=\int_{-\infty}^{\infty} f_{X}(x \mid \theta) c(\theta) \mathrm{d} \theta
$$

Again these are not PDFs. Corresponding lower and upper predictive CDFs for $X$ are derived as in (1). The predictive densities are updated by replacing $\ell(\theta)$ and $u(\theta)$ by $\ell(\theta \mid \mathbf{x})$ and $u(\theta \mid \mathbf{x})$.

In the classical Bayesian framework the essential concept is coherence. ${ }^{1}$ An analogous role is played by the generalized coherence within this new framework, according to Walley $^{4}$ (section 2.5). Coherence of the imprecise CDFs follows from the fact that they are nondecreasing functions with $0 \leq \mathscr{F}(\theta) \leq \overline{\mathscr{F}}(\theta) \leq 1$ for all $\theta$ (analogously for the other defined CDFs, Walley, ${ }^{4}$ section 4.6).

For practical application of the theory statistical models are needed. In the first place guidelines for elicitation and combination of expert opinions are required, together with methods for assessment of imprecise prior densities based on such subjective information. ${ }^{4,24}$ Here it is essential that experts are asked to give only lower and upper probabilities for observable events such as $X \leq x$, and not for parameters or for higher order probabilities. In communication with experts it is best to restrict discussion to observables, in case the underlying model (including parameters) and the mathematics are not fully understood. We think that the theory in this paper can be applied without problems in elicitation that will appear when asking precise probabilities, or even worse, higher order probabilities or possibilities that have vague theoretical interpretations and may lead to assessments by the experts without understanding what they are doing. Secondly, the idea of conjugate priors can be extended. In the usual approach conjugate priors simplify the description of the updating, and their parameters often also correspond to fictitious data. Coolen ${ }^{25,26}$ has proposed imprecise conjugate prior densities to allow these benefits to be incorporated in the approach through imprecise priors.

In Section 4 two examples using imprecise conjugate priors are given. When these extensions are used in Bayesian decision theory, the expected loss for a particular decision is also imprecise. The standard Bayesian model with a precise prior yields a single value for the expected loss; in the present case it seems that all possible losses corresponding to all possible priors have to be calculated for each decision. Coolen ${ }^{27}$ shows that few calculations are required to derive the smallest interval containing all possible values of the expected loss corresponding to a decision. To determine an optimal decision the intervals for the expected losses have to be compared with the help of an extra criterion, for example a minimax rule. Thus while there is a little more to be done, the technique avoids an unsatisfactory aspect of classical Bayesian decision theory in which an expected loss corresponding to a possible decision can be calculated exactly, while the prior is based on little information (Walley, ${ }^{4}$ section 5.6). Here the impor- 
tant difference between stochastic uncertainty and pure lack of knowledge has entered the theory, and in practical application this leads to quite logical results (for an example see Coolen ${ }^{28}$ ).

Central to the theory is the concept of imprecision which relates to the value attributed to the available information on which subjective probability statements about an event are based. The imprecision changes as new data update the prior and predictive distributions. These changes in imprecision need to be considered and described by models which are reasonably in accord with intuition. Coolen ${ }^{29}$ in an analysis of Bernoulli experiments, shows that quite simple models can easily respond correctly to cases as prior-data conflict (new data contradict prior information, Walley, ${ }^{4}$ section 5.4). Most statistical theories, Bayesian included, cannot deal with prior-data conflict because the value one adds to the available information, on which the probability statements are based, cannot be represented by imprecision, as it should be (think about the interpretation of imprecise probabilities). From this point of view the generalization of the classical precise concept of probabilities is necessary to deal correctly with different sorts of information. It seems to be logical that, in the case that new information contradicts old information, the value one adds to the total amount of information might decrease, modelled by increasing imprecision.

\section{EXAMPLES}

We illustrate the possibilities of the concept by two simple examples that are of interest in the theory of reliability.

\section{Example 1}

In a reliability test the reliability is to be estimated by testing 10 items for a predetermined time $t$. The reliability at time $t$ is then $\mathscr{R}(t)$ and we have a binomial situation with $n=10$ and $\pi=\mathscr{R}(t)$. Thus our statements about $\mathscr{R}(t)$ are based on statements about the parameter $\pi$ in the binomial distribution $\operatorname{Bin}(10, \pi){ }^{29}$ Statements about future values of the number of failures in a time period $t$ are based on predictive distributions. In an experiment we observe that $x$ items survive the test, and $10-x$ have failed; thus the probability mass function for the number of failed items is just

$$
P_{X}(X=x \mid \pi)=\left(\begin{array}{c}
10 \\
x
\end{array}\right) \pi^{x}(1-\pi)^{10-x}
$$

for

$$
x \in\{0,1, \ldots, 10\} \text { and } 0 \leq \pi \leq 1
$$

In analysing data derived from such a reliability test suppose that the imprecise densities for $\pi$ are derived from the beta family: ${ }^{24}$

$$
\begin{gathered}
\ell_{\pi}(p)=p(1-p)^{7} / \mathbf{B}(2,8) \\
u_{\pi}(p)=\ell_{\pi}(p)+c_{0} p^{3}(1-p)^{5} / \mathbf{B}(4,6),
\end{gathered}
$$

and

$$
0 \leq p \leq 1
$$

where we suppose $c_{0}=1$. If data $(n, s)$ become available, where $n$ is the total number of data (assumed to be independent) and 1 the number of successes, then the likelihood is

$$
\mathscr{L}_{X}(X=\downarrow \mid \pi)=\left(\begin{array}{c}
10 \\
s
\end{array}\right) \pi(1-\pi)^{10-\lrcorner}
$$

\begin{tabular}{|c|c|c|c|c|c|c|c|c|c|c|c|c|}
\hline \multirow[b]{2}{*}{$x$} & \multicolumn{4}{|c|}{ Prior } & \multicolumn{4}{|c|}{ Posterior $(10,3)$} & \multicolumn{4}{|c|}{ Posterior $(10,8)$} \\
\hline & $\mathscr{F}_{X}$ & $\overline{\mathscr{F}}_{X}$ & $\mathscr{P}_{X}$ & $\overline{\mathscr{P}}_{\boldsymbol{X}}$ & $\mathscr{F} x$ & $\overrightarrow{\mathscr{F}}_{X}$ & $\mathscr{P}_{\boldsymbol{X}}$ & $\overline{\mathscr{P}}_{\boldsymbol{X}}$ & $\mathscr{F}_{X}$ & $\bar{F}_{X}$ & $\mathscr{\mathscr { P }}_{X}$ & $\overline{\mathscr{P}}_{\boldsymbol{X}}$ \\
\hline 0 & $0 \cdot 1070$ & 0.2354 & $0 \cdot 1070$ & 0.2354 & 0.0628 & $0 \cdot 1144$ & 0.0628 & $0 \cdot 1144$ & 0.0007 & 0.0098 & 0.0007 & 0.0098 \\
\hline 1 & 0.2436 & 0.5159 & $0 \cdot 1294$ & $0 \cdot 3077$ & 0.2014 & 0.3524 & $0 \cdot 1344$ & $0 \cdot 2485$ & 0.0046 & 0.0679 & 0.0038 & 0.0588 \\
\hline 2 & 0.3831 & 0.7355 & $0 \cdot 1123$ & 0.3057 & 0.3874 & 0.6112 & $0 \cdot 1623$ & $0 \cdot 3096$ & 0.0155 & $0 \cdot 2309$ & 0.0107 & $0 \cdot 1770$ \\
\hline 3 & 0.5196 & $0 \cdot 8712$ & 0.0813 & $0 \cdot 2732$ & 0.5837 & 0.8037 & $0 \cdot 1396$ & $0 \cdot 2898$ & 0.0379 & 0.4841 & 0.0209 & 0.3443 \\
\hline 4 & 0.6514 & 0.9431 & 0.0509 & $0 \cdot 2280$ & 0.7565 & 0.9151 & 0.0925 & 0.2227 & 0.0767 & 0.7189 & 0.0312 & 0.4947 \\
\hline 5 & 0.7729 & 0.9773 & 0.0278 & $0 \cdot 1771$ & 0.8818 & 0.9687 & 0.0489 & $0 \cdot 1437$ & $0 \cdot 1399$ & $0 \cdot 8692$ & 0.0369 & 0.5842 \\
\hline 6 & 0.8736 & 0.9921 & $0 \cdot 0133$ & $0 \cdot 1252$ & 0.9543 & 0.9904 & 0.0210 & 0.0768 & $0 \cdot 2434$ & 0.9467 & 0.0340 & 0.6071 \\
\hline 7 & 0.9437 & 0.9977 & 0.0054 & 0.0775 & 0.9866 & 0.9977 & 0.0072 & 0.0330 & 0.4150 & 0.9815 & $0 \cdot 0236$ & 0.5670 \\
\hline 8 & 0.9819 & 0.9995 & 0.0018 & 0.0395 & 0.9973 & 0.9996 & 0.0019 & $0 \cdot 0108$ & 0.6684 & 0.9951 & 0.0119 & 0.4594 \\
\hline 9 & 0.9968 & 0.9999 & 0.0004 & 0.0149 & 0.9997 & $1 \cdot 0000$ & 0.0003 & 0.0024 & 0.9097 & 0.9993 & 0.0041 & 0.2819 \\
\hline 10 & 1 & 1 & $0 \cdot 0001$ & 0.0032 & 1 & 1 & 0.0000 & 0.0003 & 1 & 1 & 0.0007 & 0.0903 \\
\hline
\end{tabular}

and the imprecise posterior densities for $\pi$ are:

$$
\ell_{\pi}(p \mid n, \jmath)=p^{1+s}(1-p)^{7+n-s} / \mathbf{B}(2,8)
$$

and

$\left.\left.u_{\pi}(p \mid n\lrcorner,\right)=\ell_{\pi}(p \mid n\lrcorner,\right)+c_{n} p^{3+\jmath}(1-p)^{5+n-\jmath} / \mathbf{B}(4,6)$,

Table 1. Imprecise predictive cdf's and probabilities of $X$ in example 1 


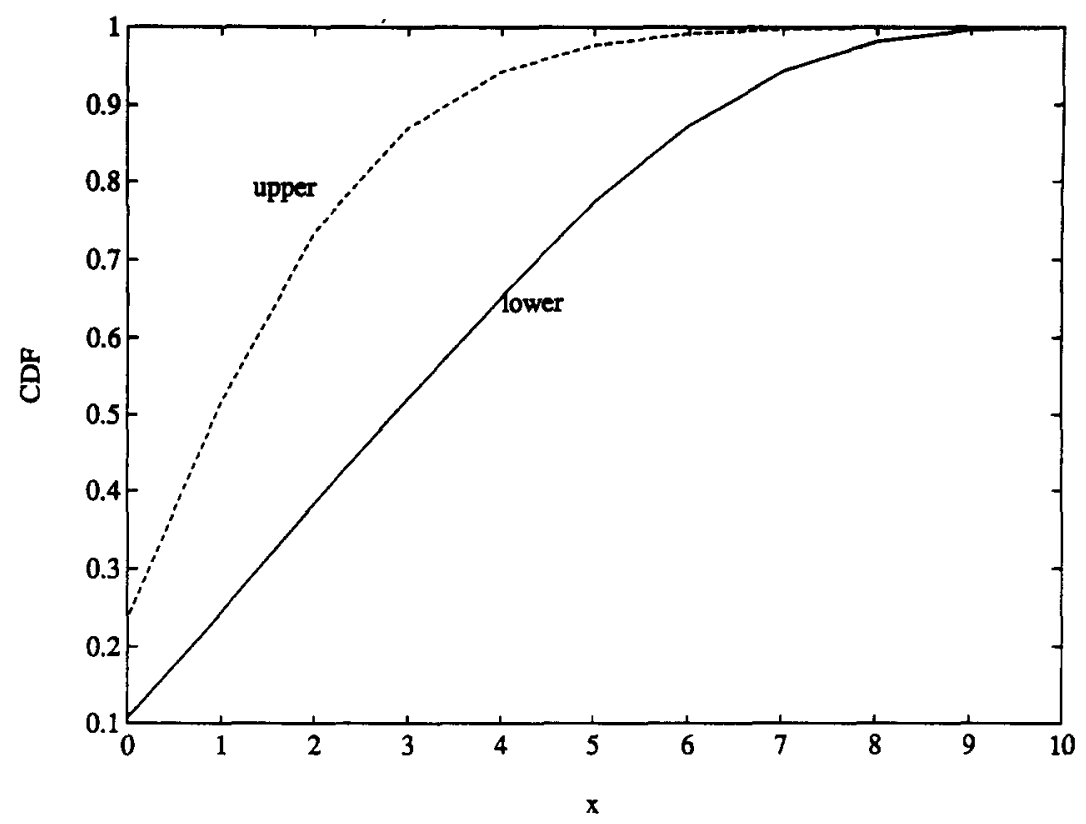

Fig. 1. Imprecise prior predictive CDFs.

with

$$
c_{n}=(1+n / 10)^{-1}
$$

This model is such that, according to Walley's measure of information, the value of prior subjective information to the decision-maker is equivalent to the value of 10 observations. Table 1 and Figs 1-6 give the imprecise predictive CDFs and probabilities of $X$, corresponding to the imprecise priors and posteriors for two cases. The first posterior is based on data $(10,3)$, that seem to be reasonably in agreement with the prior information. The second posterior is based on data $(10,8)$, that are unlikely under the prior information. Thus in one case the data play a confirmatory role, and in the other there is conflict between the prior and the data. The conflict can be seen in the increase in the imprecision in the second case. Moreover, the value the decision-maker adds to the total information decreases in the case of conflict. This effect is clear on looking at Figs 1-6, representing the numbers of Table 1 . Figures 1-3 show predictive CDFs, that is imprecise probabilities for the events $X \leq x$, and Figs 4-6 show predictive imprecise probabilities for the events $X=x$; these

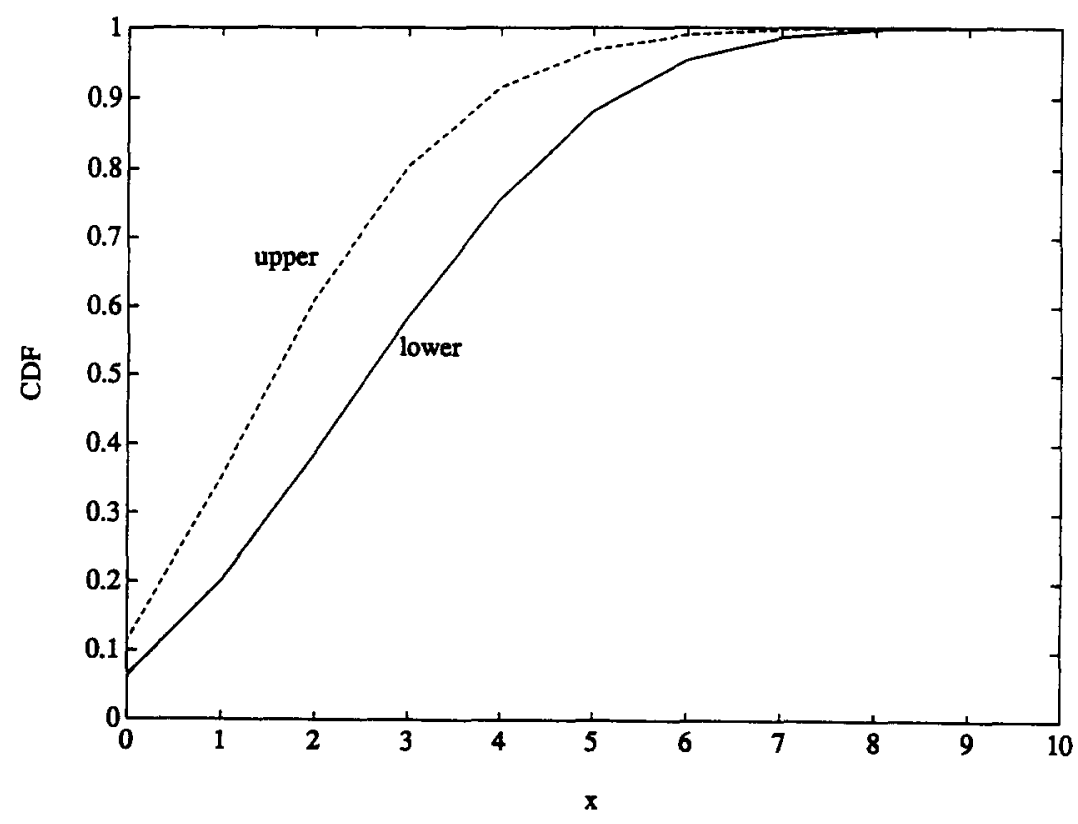

Fig. 2. Imprecise posterior predictive CDFs, data $(10,3)$. 


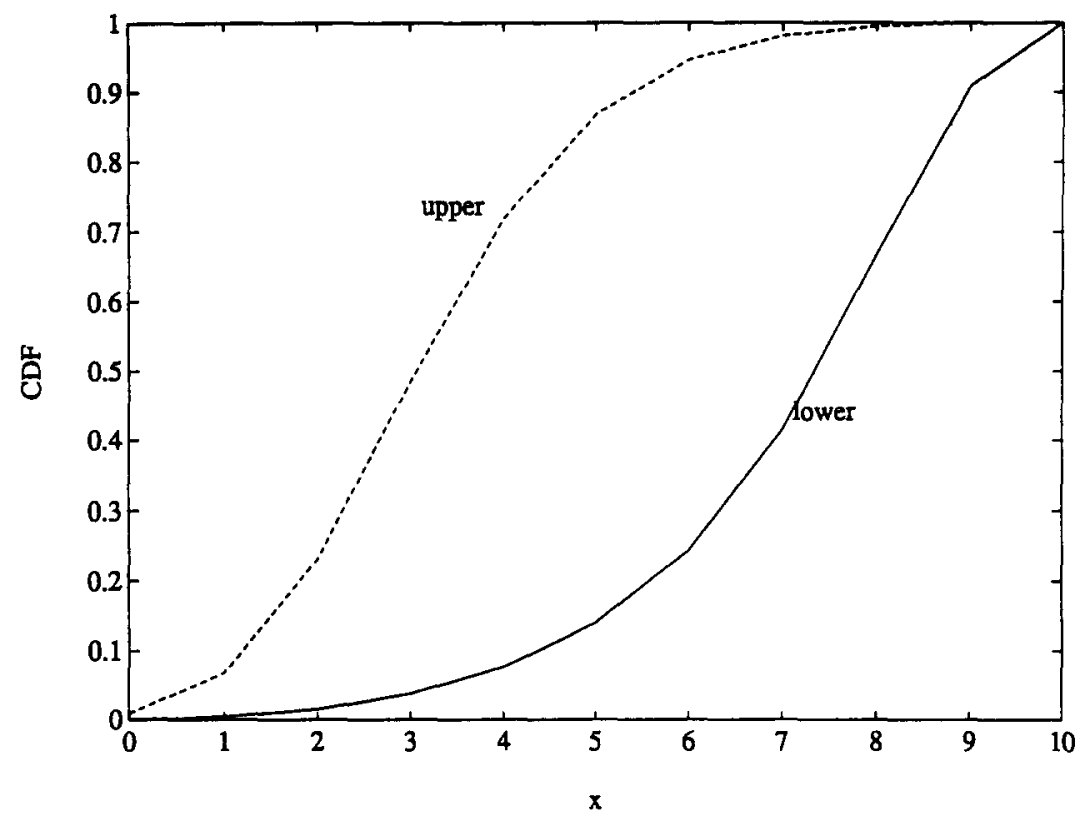

Fig. 3. Imprecise posterior predictive CDFs, data $(10,8)$.

figures should be reasonably well understood by non-mathematicians.

\section{Example 2}

Suppose that we are interested in the lengths of intervals between breakdowns of a system, which follows a Poisson process; thus the intervals are independent and identically exponentially distributed. We consider the problem of determining a distribution for the length of the intervals between failures. To show how the idea of conjugate priors can be maintained we express the prior knowledge in terms of imprecise conjugate priors. ${ }^{24}$ In this case the distribution of the length, $X$, of intervals is the exponential

$$
f_{X}(x \mid \lambda)=\lambda \mathrm{e}^{-\lambda x}, \quad x \geq 0 \text { and } \lambda>0
$$

Since the density is exponential, the natural conjugates come from the gamma family. Thus suppose the imprecise prior densities for $\lambda$ are:

$$
\ell_{\lambda}(l)=l \mathrm{e}^{-l}
$$

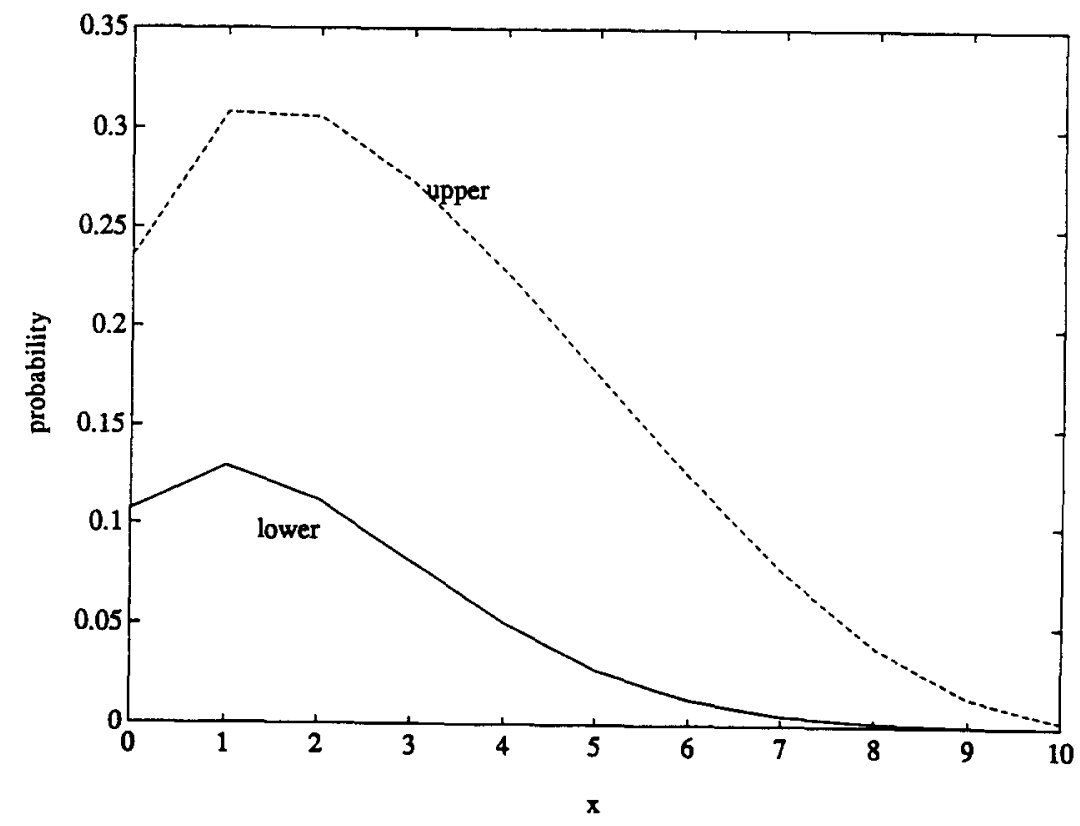

Fig. 4. Imprecise prior predictive probabilities. 


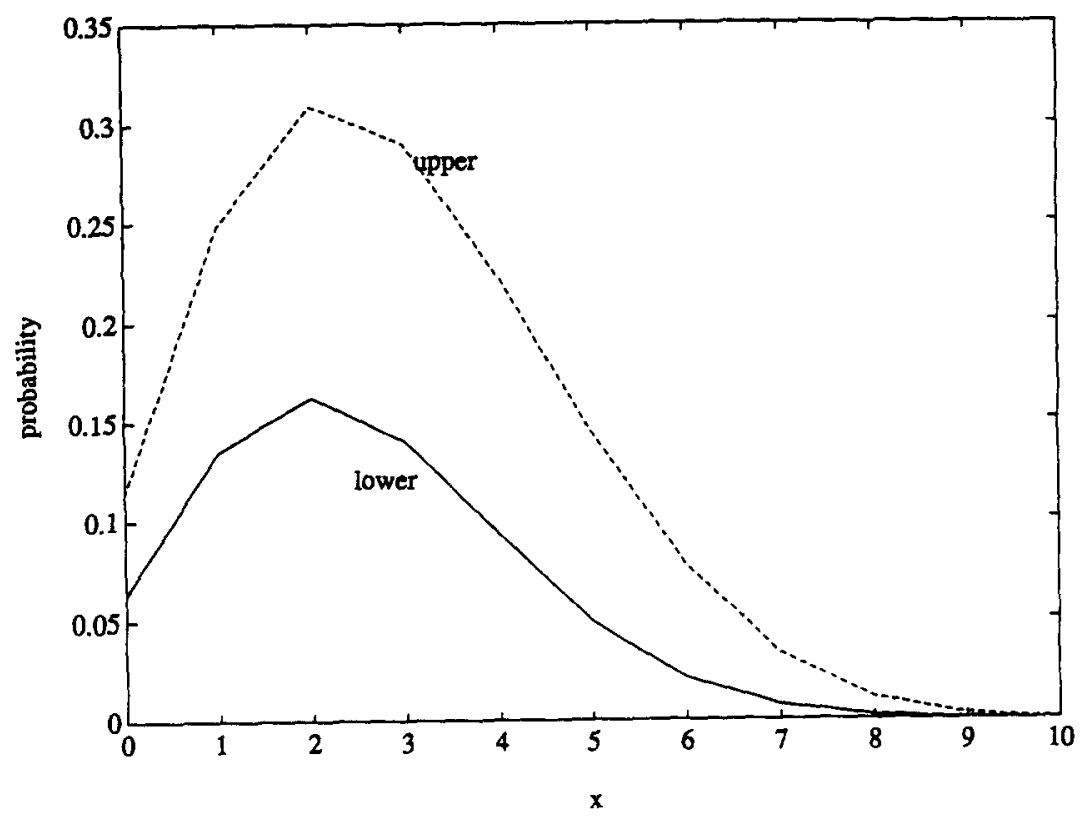

Fig. 5. Imprecise posterior predictive probabilities, data $(10,3)$.

and

$$
u_{\lambda}(l)=\ell_{\lambda}(l)+c_{0} 4 l \mathrm{e}^{-2 l}, \quad \text { with } c_{0}=1 \quad(l \geq 0)
$$

Littlewood's $\mathrm{s}^{30}$ software reliability model can be generalized in a similar way if we assume that the mixing distributions are given imprecisely in the gamma family.

If $n$ independent data $\left\{x_{1}, \ldots, x_{n}\right\}$ become available, sufficient statistics are $(n, t)$, with $t=$ $\sum_{i=1}^{n} x_{i}$, and the imprecise posterior densities for $\lambda$ are:

$$
\ell_{\lambda}(l \mid n, t)=(1+t)[(1+t) l]^{1+n} \mathrm{e}^{-(1+f)} / \Gamma(2+n)
$$

and

$u_{\lambda}(l \mid n, t)$

$$
=\ell_{\lambda}(l \mid n, t)+c_{n}(2+t)[(2+t) l]^{1+n} \mathrm{e}^{-(2+n l} / \Gamma(2+n)
$$

with $c_{n}=(1+n)^{-1}$. The value of $c_{n}$ is such that the value of the subjective prior information is equivalent to that of one observation. In Figs 7 and 8 the imprecise predictive reliability functions for $X$ and the corresponding hazard rates are given, together with the same functions for the imprecise posteriors derived after two observations summing to 2 have become available.

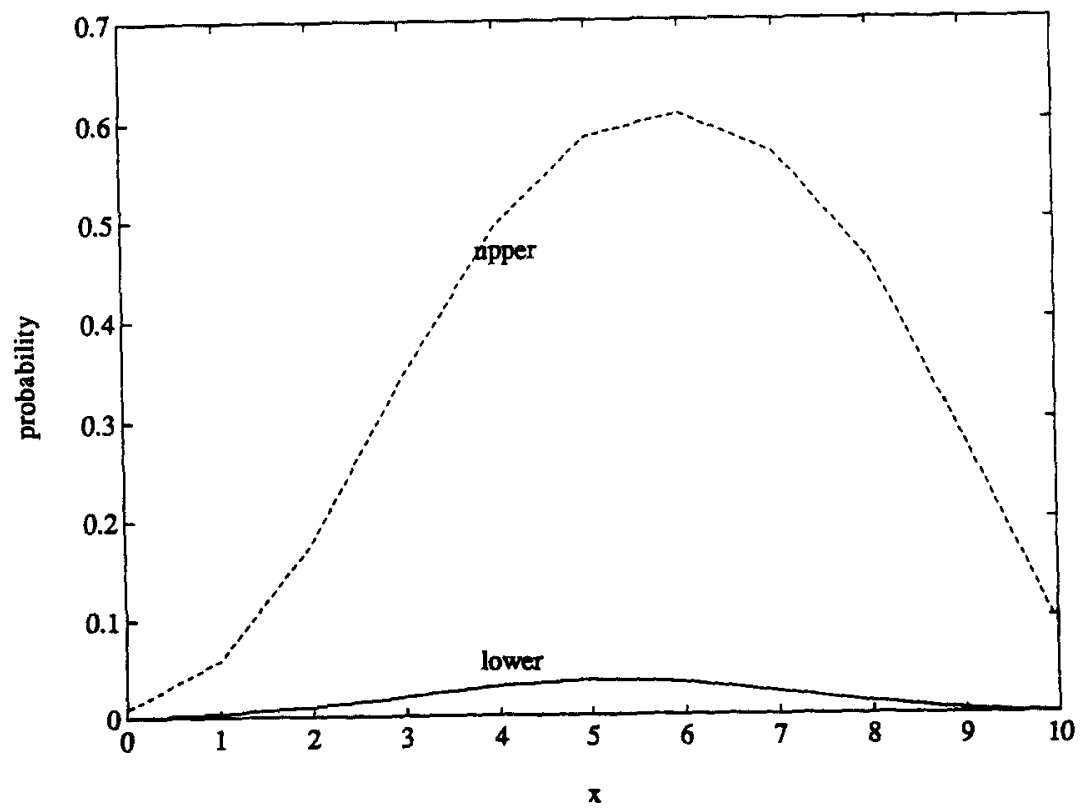

Fig. 6. Imprecise posterior predictive probabilities, data $(10,8)$. 


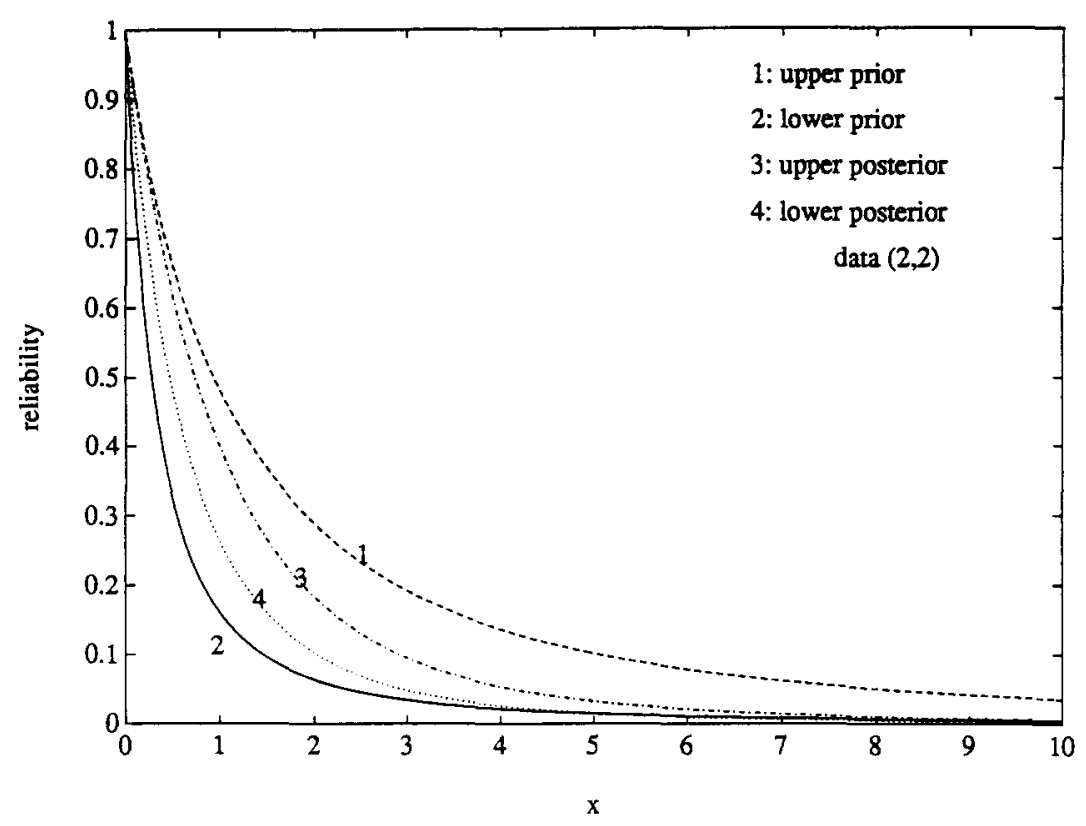

Fig. 7. Imprecise predictive reliability functions.

The same functions are given in Figs 9 and 10, but this time with posteriors derived from two observations summing to 5 . This second case is an example of data that are in conflict with the prior thoughts, although very little value was added to the subjective opinions.

\section{CONCLUSIONS AND ADDITIONAL REMARKS}

Thus, we have shown how the commonly used concepts in reliability theory can be extended in a sensible way and combined with prior knowledge through the use of imprecise probabilities. Little more is demanded than in a standard Bayesian approach, and in return some of the difficulties of the standard approach are avoided. In particular, the use of imprecise priors offers better ways of eliciting and expressing expert knowledge. At the same time, there is a calculus of imprecise probabilities ${ }^{24}$ which ensures that the expert opinions are consistently expressed and combined. The interpretation of results requires a little more of users since now all measures of interest are given as lying in a range between a lower and

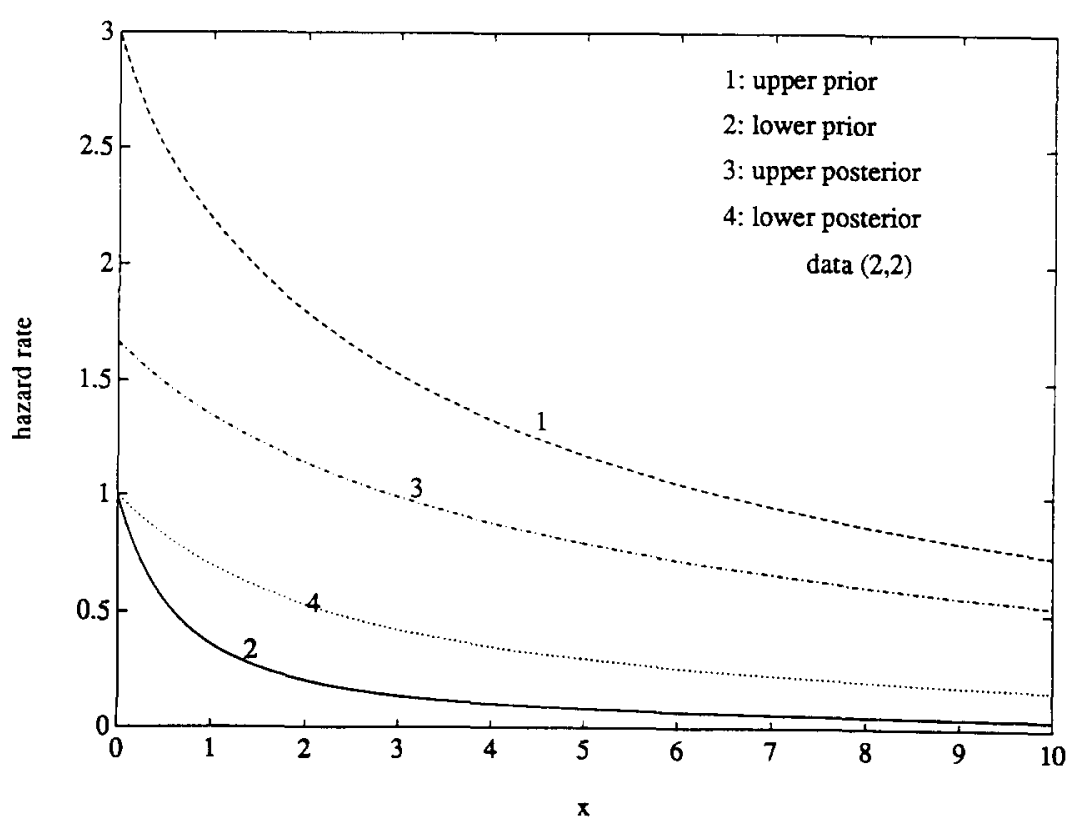

Fig. 8. Imprecise predictive hazard rates. 


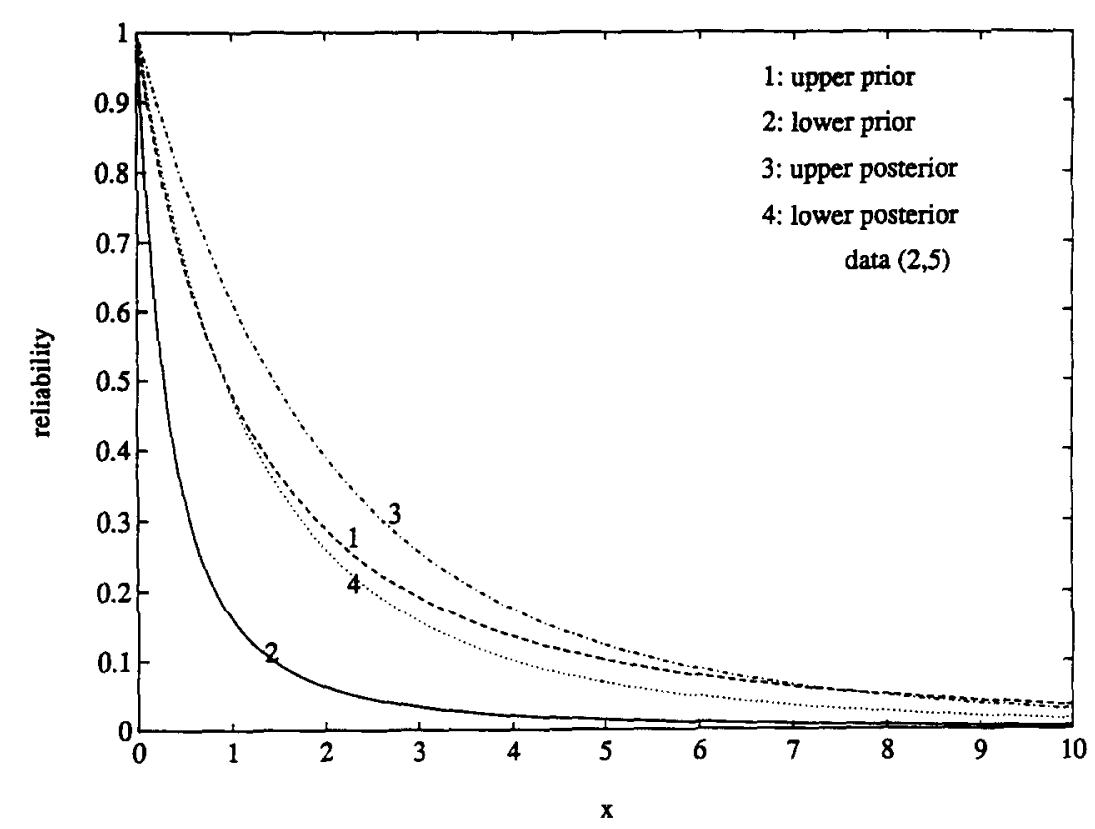

Fig. 9. Imprecise predictive reliability functions.

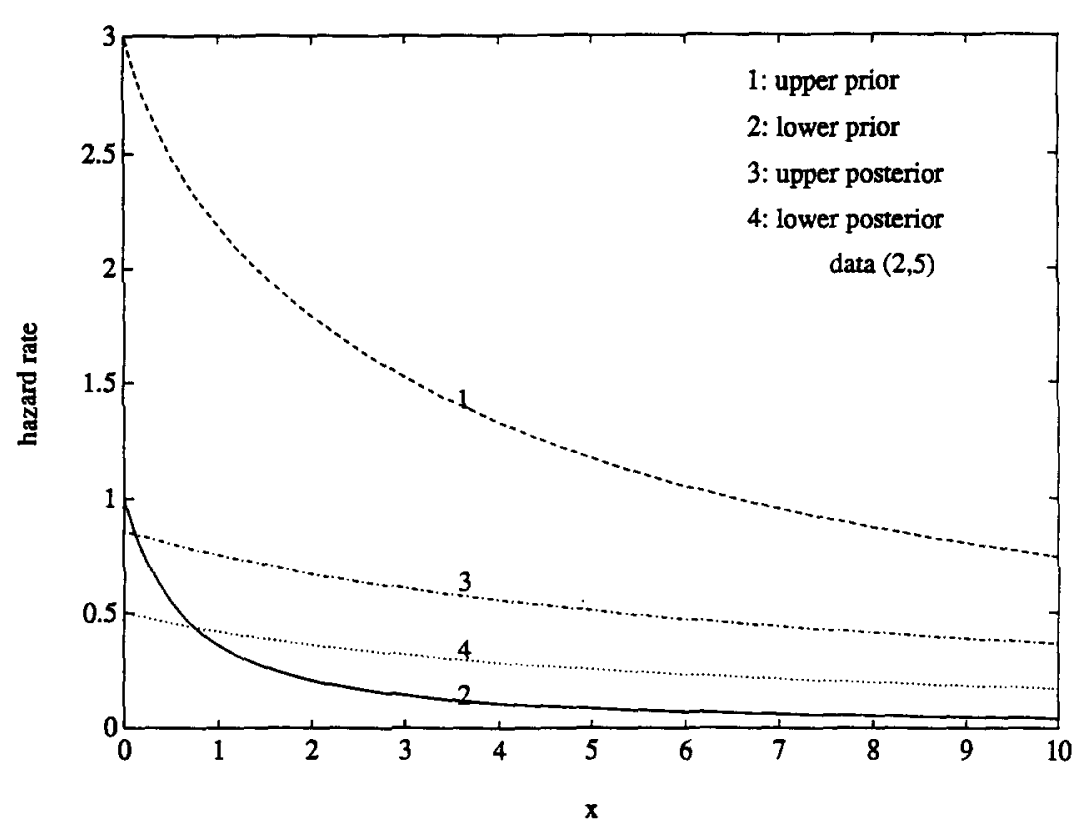

Fig. 10. Imprecise predictive hazard rates.

upper value. ${ }^{28}$ However, as we have seen the range can be an increasing or decreasing function of the amount of data; when the data are consistent with the preceding data and with the prior opinions, the ranges decrease; when the latest data are not consistent with the current state of knowledge, the range can increase. Thus the intervals provide through the measures of imprecision an indication of changes in the object of study.
The concepts described here, and other recent alternatives to the classical probability theory, need further testing in practical application, which may also lead to new questions and ideas.

\section{ACKNOWLEDGEMENTS}

The authors would like to thank an anonymous referee for remarks leading to better presentation, 
and the editor-in-chief, G. E. Apostolakis, for making us aware of some other similar developments in the literature.

\section{REFERENCES}

1. Lindley, D. V., The present position in Bayesian statistics (1988 Wald memorial lectures, with discussion). Statistical Science 5(1) (1990) 44-89.

2. Martz, H. F. \& Waller, R. A., Bayesian Reliability Analysis. Wiley, New York, 1982.

3. Sander, P. \& Badoux, R. (eds), Bayesian Methods in Reliability. Kluwer Academic Publishers, Dordrecht, 1991.

4. Walley, P., Statistical Reasoning with Imprecise Probabilities. Chapman \& Hall, London, 1991.

5. Cooke, R. M., Experts in Uncertainty. Oxford University Press, Oxford, 1991.

6. Coolen, F. P. A., Mertens, P. R. \& Newby, M. J., A Bayes-competing risk model for the use of expert judgement in reliability estimation. Reliability Engineering and System Safety, 35 (1992) 23-30.

7. Dubois, D. \& Prade, H., On the relevance of non-standard theories of uncertainty in modeling and pooling expert opinions. Reliability Engineering and System Safety, 36 (1992) 95-107.

8. Chun, M. \& Ahn, K., Assessment of the potential applicability of fuzzy set theory to accident progression event trees with phenomenological uncertainties. Reliability Engineering and System Safety, 37 (1992) 237-52.

9. Rao, S. S. \& Dhingra, A. K., Reliability and redundancy apportionment using crisp and fuzzy multiobjective optimization approaches. Reliability Engineering and System Safety, 37 (1992) 253-61.

10. De Finetti, B., Theory of Probability (vols 1 and 2). Wiley, New York, 1974.

11. Boole, G., An Investigation of the Laws of Thought on which are Founded the Mathematical Theories of Logic and Probabilities. Macmillan, London, 1854; reprinted by Dover, 1958 .

12. Smith, C. A. B., Consistency in statistical inference and decision (with discussion). Journal of the Royal Statistical Society, series B, 23 (1961) 1-25.

13. Good, I. J., Subjective probability as the measure of a non-measurable set. In Logic, Methodology and Philosophy of Science, eds E. Nagel, P. Suppes \& A. Tarski. Stanford University Press, 1962, pp. 319-29.

14. Dempster, A. P., A generalization of Bayesian inference (with discussion). Journal of the Royal Statistical Society, series B, 30 (1968) 205-47.

15. Walley, P. \& Fine, T. L., Varieties of model (classificatory) and comparative probability. Synthese, 41 (1979) 321-74.

16. Wolfenson, M. \& Fine, T. L., Bayes-like decision making with upper and lower probabilities. Journal of the American Statistical Association, 77(377) (1982) 80-8.

17. Walley, P. \& Fine, T. L., Towards a frequentist theory of upper and lower probability. Annals of Statistics, 10(3) (1982) 741-61.

18. DeRobertis, L. \& Hartigan, J. A., Bayesian inference using intervals of measures. Annals of Statistics, 9 (1981) 235-44.

19. Pericchi, L. R. \& Walley, P., Robust Bayesian credible intervals and prior ignorance. International Statistical Review, 58(1) (1991) 1-23.

20. Berger, J., Statistical Decision Theory and Bayesian Analysis. Springer-Verlag, New York, 1985.

21. Berger, J. \& Berliner, L. M., Robust Bayes and empirical Bayes analysis with $\varepsilon$-contaminated priors. Annals of Statistics, 14(2) (1986) 461-86.

22. Meczarski, M. \& Zielinski, R., Stability of the Bayesian estimator of the poisson mean under the inexactly specified gamma prior. Statistics \& Probability Letters, 12 (1991) 329-33.

23. Walley, P., Measures of uncertainty in expert systems. Research Report, April 1991/27, the University of Western Australia, Australia, 1991.

24. Coolen, F. P. A., Elicitation of expert knowledge and assessment of imprecise prior densities for lifetime distributions. Cosor-Memorandum 92-12, Eindhoven University of Technology, 1992.

25. Coolen, F. P. A., Maximum imprecision related to intervals of measures and Bayesian inference with imprecise conjugate prior densities. CosorMemorandum 92-07, Eindhoven University of Technology, 1992.

26. Coolen, F. P. A., Imprecise conjugate prior densities for the one-parameter exponential family of distributions. Statistics \& Probability Letters, 16 (1993) 337-42.

27. Coolen, F. P. A., Bounds for expected loss in Bayesian decision theory with imprecise prior probabilities. Cosor-Memorandum 92-40, Eindhoven University of Technology, 1992 The Statistician (1994).

28. Coolen, F. P. A., Bayesian decision theory with imprecise prior probabilities applied to replacement problems. Cosor-Memorandum 93-09, Eindhoven University of Technology, 1993 Proceedings of the 1993 SRE-Symposium, October 1993, the Netherlands.

29. Coolen, F. P. A., On Bernoulli experiments with imprecise prior probabilities, Cosor-Memorandum 9219. Eindhoven University of Technology, 1992 The Statistician (1994).

30. Littlewood, B., Forecasting software reliability. In Bayesian Methods in Reliability, eds P. Sander \& R. Badoux. Kluwer Academic Publishers, Dordrecht, 199. pp. 135-201. 